\title{
Construção de um Rastreador Solar de dois
}

Guimarães, L. B.

Escola Politécnica de Pernambuco Universidade de Pernambuco 50.720-001 - Recife, Brasil lorena_bguimaraes@yahoo.com.br
Malagón, L. A. G.

Escola Politécnica de Pernambuco

Universidade de Pernambuco

50.720-001 - Recife, Brasil

lagomezma@poli.br

Resumo Em decorrência dos impactos ambientais provenientes do crescimento vertiginoso da emissão de gases e partículas poluentes, a busca por novas formas de captação de energia tem-se intensificado. Dentre essas fontes de energia a que ganha maior destaque é a solar, por ser uma fonte limpa e inesgotável. Há duas maneiras de se obter a energia solar: a térmica ou a fotovoltaica. A obtenção da fotovoltaica é feita com o uso de painéis solares, ou seja, por meio de superfícies de captação. Essas superfícies podem ser fixas, de um ou dois eixos. Algumas pesquisas realizadas nessa área demonstram que o rastreador solar de dois eixos melhora quantitativamente e qualitativamente o aproveitamento do potencial energético oferecido pelo sol. Sendo assim, esse projeto de pesquisa visa à confecção de um seguidor solar de dois eixos a fim de possibilitar a obtenção de maiores conhecimentos a cerca desse campo de atuação.

Abstract As a result of environmental impacts arising from the rapid growth of the emission of gaseous and particulate pollutants, the search for new forms of energy harvesting has intensified. Among these sources of energy that the spotlight is solar, as a clean and inexhaustible source. There are two ways to get solar energy: thermal or photovoltaic. Obtaining the photovoltaic is made with the use of solar panels, or by means of catchment surfaces. These surfaces can be fixed in one or two axes. Some research conducted in this area show that the two-axis solar tracker improves quantitatively and qualitatively, the use of the potential offered by the sun. Thus, this research project aims at making a two-axis solar tracker to allow obtaining more knowledge about this field. 


\section{Introdução}

Desde quando o Homem percebeu o movimento aparente do sol e desenvolveu os primeiros relógios solares ainda na era pré-histórica, ficou cada vez mais clara a influência do sol no seu cotidiano. Com o passar dos anos, o advento da ciência nessa área proporcionou em 1839 a observação, pela primeira vez, do efeito fotoelétrico pelo físico francês Alexandre Becquerel, e logo após essa descoberta, em 1883, Charles Fritts, conseguiu construir a primeira célula solar [1]. Assim sendo, a energia solar começou a ser explorada com mais intensidade e várias linhas de pesquisa foram criadas e baseadas na melhor maneira de se obter uma eficiência no seu potencial energético.

A energia solar é definida como a energia proveniente do sol, que pode ser tanto térmica como luminosa. Vários processos de captação foram criados desde o mais simples, como a utilização de garrafas PET para o aquecimento de água em caixas de água, até o mais complexo, como a utilização de seguidores solares de dois eixos, que possibilitam a obtenção de radiação solar de forma que os raios incidem sempre em sua superfície de forma ortogonal, assim, aproveitando a máxima potência oferecida pelo sol em certas superfícies.

Os seguidores solares são dispositivos de rastreios que dependem quase que exclusivamente da incidência dos raios solares, contudo fatores externos como condições climáticas, latitude do local de instalação, poeira, estações do ano, entre outros, podem influenciar significativamente na exploração em larga escala deste recurso. Então, várias práticas são elaboradas a fim de minimizar os efeitos desses fatores sobre o sistema de captação.

Basicamente existem dois tipos de rastreadores solares, $\mathrm{o}$ de um eixo e o de dois eixos.

Estimasse que esses seguidores possam aumentar em cerca de $20 \%$ e $35 \%$ respectivamente, a maximização da prospecção da radiação solar incidente [2]. Logo, esse projeto almeja esclarecer, através de algumas simulações, algoritmos de segmento, entre outras técnicas, determinar de forma efetiva o modelamento mais próximo do real possível, levando em conta todas as particularidades oferecidas pelo meio ao qual o sistema será exposto, a fim de proporcionar as melhores condições para que haja um avanço quanto ao empreendimento comercial energético desse sistema.

\section{Objetivos}

Basicamente existem dois tipos de rastreadores solares, o de um eixo e o de dois eixos. Estimasse que esses seguidores possam aumentar em cerca de $20 \%$ e $35 \%$ respectivamente, a maximização da prospecção da radiação solar incidente [2]. Logo, esse projeto almeja esclarecer, através de algumas simulações, algoritmos de segmento, entre outras técnicas, determinar de forma efetiva $o$ modelamento mais próximo do real possível, levando em conta todas as particularidades oferecidas pelo meio ao qual o sistema será exposto, a fim de proporcionar as melhores condições para que haja um avanço quanto ao empreendimento comercial energético desse sistema.

\subsection{Objetivos Gerais}

1. Construir um seguidor solar de dois eixos.

\subsection{Objetivos Específicos}

1. Realizar o projeto mecânico de um seguidor solar de dois eixos.

2. Realizar o projeto eletrônico do controle dos motores do seguidor solar.

3. Implementar o sistema de controle do seguidor solar.

\section{Materiais e Métodos}

Inicialmente, fizemos alguns esboços a fim de obter uma ideia preliminar de como seria a estrutura, levando em conta características como: praticidade na confecção, no deslocamento da estrutura, na economia de energia durante o seu funcionamento e no orçamento para a sua confecção. Partimos, então, para análise do material que deveria ser utilizado para a sua confecção, considerando sua devida exposição a chuvas e outros intempéries, a quantidade de energia oferecida pela bateria para alimentar o sistema, os motores e outros componentes eletrônicos. Quando atingimos um consenso sobre como seria mais ou menos a estrutura, partimos para a fase do modelamento no software SOLIDWORKS. 


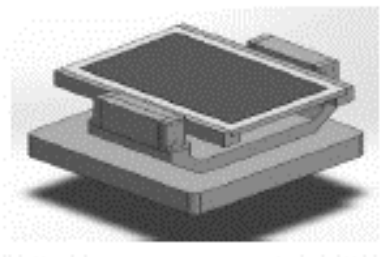

Fig 1. Primeira modelagem no SOLIDWORKS.

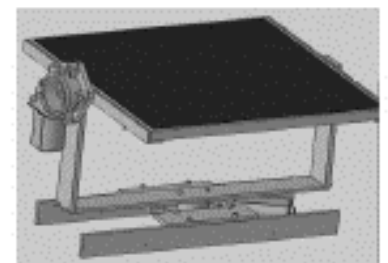

Fig 2. Segunda modelagem no SOLIDWORKS.

Chegamos então, a conclusão de que usaríamos barras e chapas de alumínio para garantir à resistência do sistema as condições climáticas a que estaria sujeito, já que o alumínio é um metal que possui alta resistência à corrosão, pois tem uma fina camada de óxido em sua superfície que o protege de oxidações, e grande leveza, por conta do seu baixo peso específico[3].

\begin{tabular}{|c|c|c|c|}
\hline Propriedades fisicas fpicas & Atrrino & AçD & Catre \\
\hline Donatess (pom?') & 2.70 & 7.86 & 896 \\
\hline 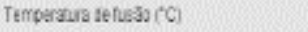 & 66) & 1500 & 11183 \\
\hline 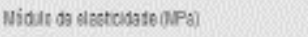 & 70000 & 205000 & 113000 \\
\hline Caescente de dirsçaso vemita ILCC: & $21.72-1$ & $11711 \cdot 6$ & $18.5,77-5$ \\
\hline 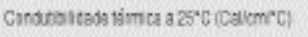 & 159 & 0.12 & ODH \\
\hline Cunaroitave elences ("suCS) & 61 & 145 & 100 \\
\hline
\end{tabular}

Tabela 1. Propriedades físicas do aluminio em comparação com o aço e o cobre.

Visando uma maior economia de energia dada pela bateria de 12 v que será acoplada para garantir o funcionamento do sistema, o motor utilizado no projeto foi um motor de vidro elétrico de carro, por se tratar de um sistema auto travante, ou seja, não há necessidade de manter a alimentação do motor para que ele permaneça em uma dada posição desejada. Em comparação com o motor de passo, motor comumente utilizado em alguns projetos de rastreadores, o motor de vidro elétrico de carro se torna mais eficaz [4]. Portanto, dois motores de vidro elétrico do tipo DSW - 3301 foram utilizados.

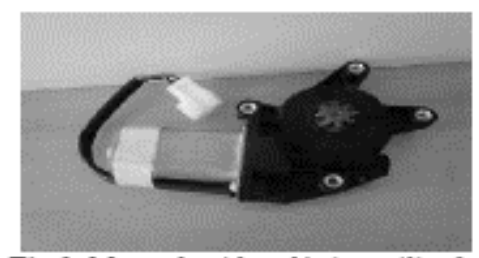

Fig 3. Motor de vidro elétrico utilizado.

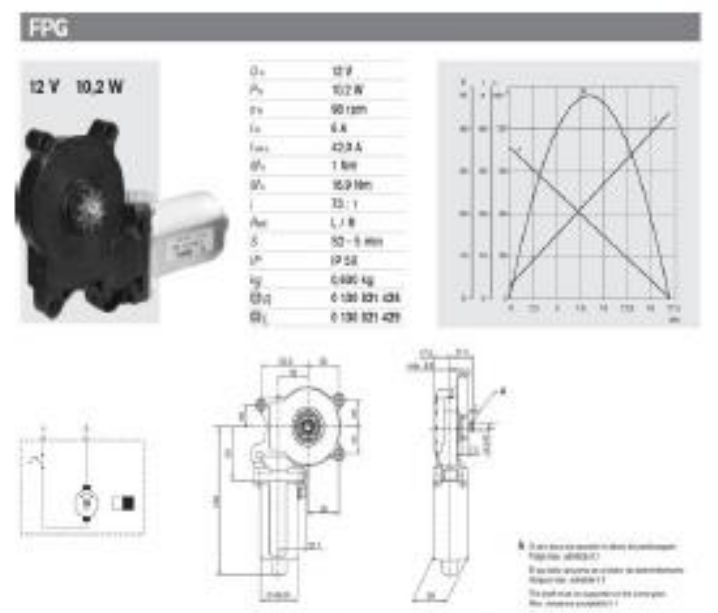

Tabela 2. Caracteristicas dos motores de vidro elétrico utilizados.

As características do painel selecionado no projeto seguem abaixo:

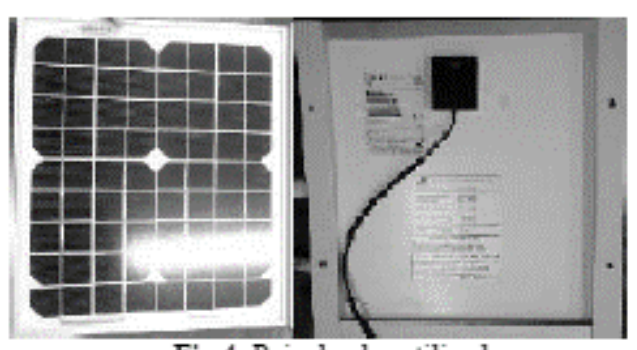

Fig 4. Painel solar utilizado.

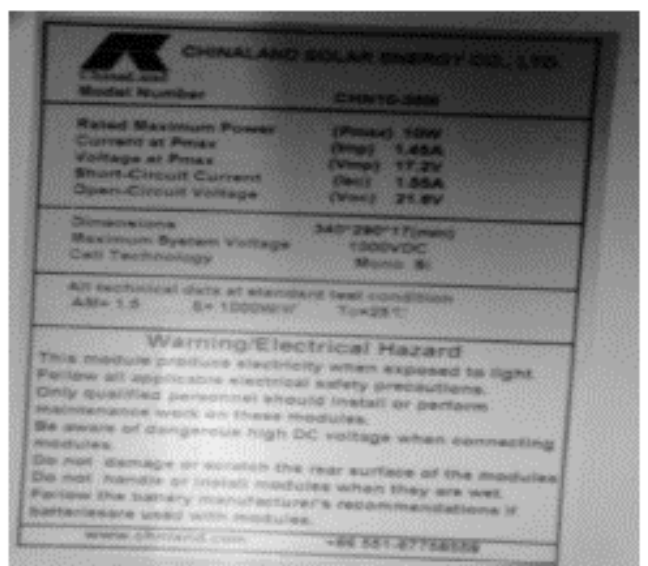

Fig 5. Características do painel solar utilizado. 
O Sistema de controle seria realizado com base nas equações da terra [5], visando o ângulo de inclinação do painel e o ângulo azimutal da superfície. O controle dos motores DC ficariam vinculados à utilização da placa de aquisição de dados ARDUINO. Dessa forma, foram compradas duas Shields IMS - 1, de modo a garantir a execução desse controle.

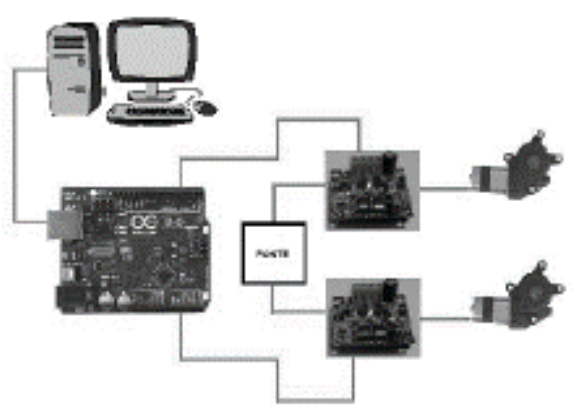

Fig 6. Detalhamento da estrutura de controle utilizada.

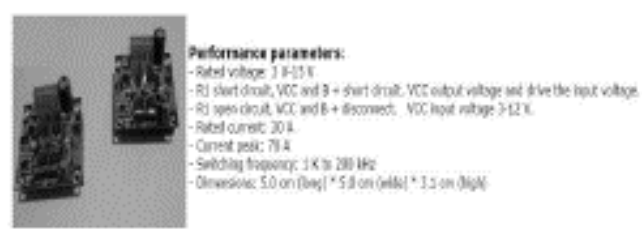

Fig 7. Detalhamento das SHIELDS IMS -1 utilizadas.

No programa de controle, as equações da terra seriam descritas, conforme foi dito anteriormente, de modo que duas variáveis seriam de maior importância, o ângulo de inclinação do painel $(\beta)$, responsável, basicamente, pela captação de radiação solar ao longo do dia, e o ângulo azimutal da superfície $(\gamma)$, responsável pela captação solar de acordo com o dia do ano, levando em conta a taxa de incidência de radiação no local aonde virá a ser instalado o seguidor de acordo com as estações do ano.

\section{$\cos \theta=\sin \delta \sin \phi \cos \beta-\sin \delta \cos \phi \sin \beta \cos \gamma+\cos \delta \cos \phi \cos \beta \cos \omega$ $+\cos \delta \sin \phi \sin \beta \cos \gamma \cos \omega+\cos \delta \sin \beta \sin \gamma \sin \omega$}

Em que,

$\Theta$ Ângulo de incidência de radiação solar.

$\delta \quad$ Ângulo de declividade solar.

$\Phi$ Latitude do local de instalação do seguidor solar.
$B$ Ângulo de inclinação do painel solar.

$\gamma \quad$ Ângulo azimutal da superfície.

$\omega$ Ângulo horário.

Essa equação é descrita com base nos critérios listados abaixo:

$$
\begin{gathered}
\cos \theta=1 \\
\gamma=\gamma_{s} \\
\beta=\theta_{z}
\end{gathered}
$$

onde, $\gamma$ s é dada por:

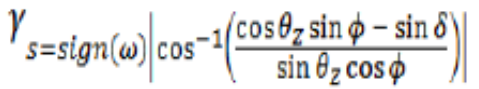

Assim, sabendo a latitude do local de instalção $(\phi)$, que tratá-se de uma variável constante, que no caso da cidade do Recife seria $08^{\circ} 03^{\prime} 14^{\prime \prime} \mathrm{S}$, e lembrando que a declividade solar e o ângulo horário serão descritos pelas equações abaixo, respectivamente:

\section{$\delta=0.006918-0.399912 \cos B+0.070257 \sin B-0.006758 \cos 2 B+0.000907 \sin 2 B$ $-0.002679 \cos 3 B+0.00148 \sin 3 B$}

$$
\omega=15\left(h_{s}-12\right)
$$

Pode-se, então, determinar o ângulo de inclinação do painel solar $(\theta z)$.

De posse desses materiais e do sistema de controle desejado, o seguidor solar pode ser construído com o auxílio da Equipe Corisco Baja e de alguns professores da Escola Politécnica de Pernambuco que forneceram embasamento teórico e prático durante o processo de confecção. O modelo foi construído no NEAR (Núcleo de Engenharia em Automação e Robótica), onde foi disponibilizada a utilização de uma fresa, de um torno e de uma furadeira, assim, as peças puderam ser usinadas, já que algumas foram torneadas e fresadas, e também puderam sofrer processos de corte. 

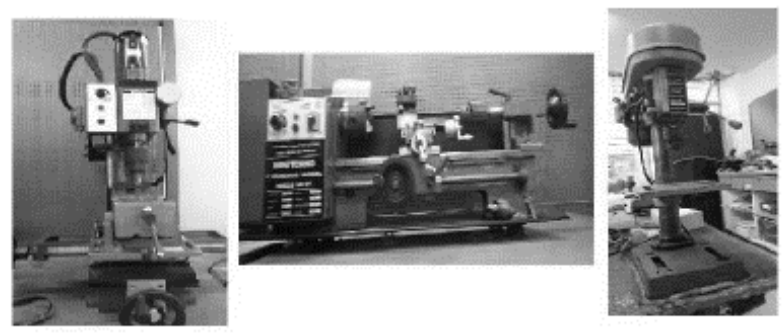

Fig 8. Representação da fresa, torno e furadeira respectivamente.

\section{Discussão e Resultados}

1. Construção do rastreador solar de dois eixos
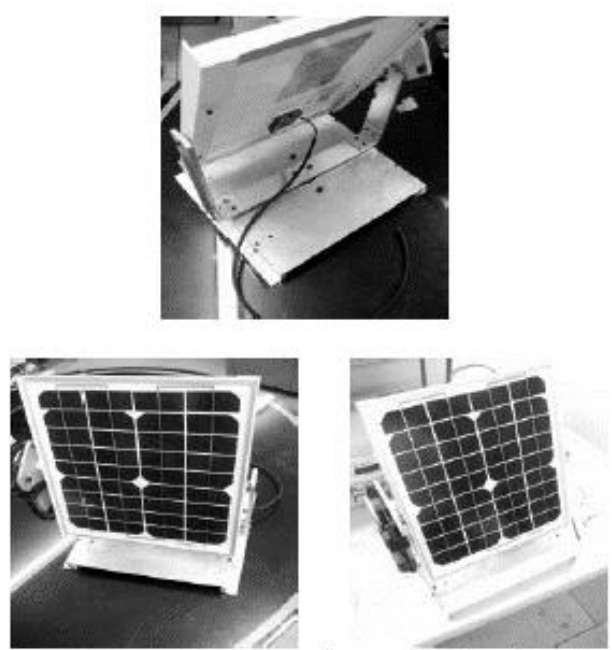

Fig 9. Vistas Projeto Finalizado.

Durante o processo construtivo do rastreador solar de dois eixos foi-se observado a complexidade exigida pelo projeto em termos econômicos, mecânicos e eletrônicos. Apesar da última fase de análise, que se tratava do controle do seguidor, ter sido impossibilitada de ser executada, por conta do longo período de tempo que levou para a chegada dos componentes eletrônicos, que infelizmente chegaram duas semanas antes da entrega do projeto, sua confecção se tornou extremamente trabalhosa. Em virtude dessa dificuldade que tivemos por conta da chegada dos componentes, pretende-se ampliar o período de trabalho desse projeto com a finalidade de obter-se o surgimento de outra linha de pesquisa em um projeto futuro, em que se deseja identificar o ângulo ótimo de inclinação de painéis solares na cidade de Recife.

\section{Conclusão}

Com base no projeto de pesquisa realizado, tem-se que à aplicação do mecanismo empregado no seguidor solar de dois eixos tem maior eficiência na obtenção de radiação, já que o mesmo possui um melhor desempenho do que as outras formas convencionais de sistemas solares fotovoltaicos de captação de radiação solar.

\section{Referências}

[1] BlueSol Educacional (http://www.bluesol.com/energia-solar/histria-rpida-da-energiasolar-fotovoltaica/ ).

[2] E. B. Pereira, F. Ramos Martins, S. Luna de Abreu, R. Rüther, Atlas Brasileiro de Energia Solar. São José dos Campos. INEP (2006)

[3] ABAL - Associação Brasileira do Alumínio (http://www.abal.org.br/aluminio/caracteristicasquimicas-e-fisicas/ ).

[4] Laboratório de Garagem (http://labdegaragem.com/forum/topics/motor-dc-de-vidro-eletrico-mabuchi).

[5] John A. Duffie \& William A. Beckman, Solar Engineering of Thermal Processes. Third edition. 